\title{
The opioid agonist codeine and antagonist naltrexone do not affect voluntary suppression of capsaicin induced cough in healthy subjects
}

\author{
H.A. Hutchings, R. Eccles
}

The opioid agonist codeine and antagonist naltrexone do not affect voluntary suppression of capsaicin induced cough in healthy subjects. H.A. Hutchings, R. Eccles. CERS Journals Ltd 1994.

ABSTRACT: Opioids exert an analgesic action by mimicking the effects of endogenous neurotransmitter substances in the central nervous system. Opioids are widely used as antitussives, and it is reasonable to assume that endogenous opioids are involved in the control of cough. In order to investigate this hypothesis, a parallel design study was carried out to examine the effects of $50 \mathrm{mg}$ codeine (opioid agonist), $50 \mathrm{mg}$ naltrexone (opioid antagonist) and placebo on capsaicin-induced cough in 80 healthy volunteers (mean age 25 yrs).

Volunteers received two capsaicin challenge units (each consisting of five inhalations of different concentrations of capsaicin, $\left.0.00-3.33 \times 10^{-4} \mathrm{M}\right)$. On one challenge unit subjects were instructed to suppress cough, and on the other challenge unit subjects coughed freely. Coughs were recorded on a tape cassette player and later played back into a pen recorder to produce integrated sound traces.

The number of coughs in the suppression challenge unit was significantly reduced in all three treatment groups compared to that recorded in the non-suppression challenge unit. Comparisons between the three treatment groups showed that there was no statistical difference between the three groups both before and $90 \mathrm{~min}$ after treatment for the total coughs in the suppression challenge unit and for the total coughs in the non-suppression challenge unit.

These results demonstrate that capsaicin-induced cough can be voluntarily suppressed, but that both suppressed and non-suppressed cough were unaffected by treatment with codeine, naltrexone or placebo. These results do not provide any support for the hypothesis that capsaicin-induced cough is influenced by endogenous opioid substances.

Eur Respir J., 1994, 7, 715-719.

Common Cold and Nasal Research Centre, Dept of Physiology, University of Wales College of Cardiff, Cardiff, UK.

Correspondence: H.A. Hutchings

Common Cold and Nasal Research Centre Department of Physiology

University of Wales College of Cardiff

P.O. Box 902

Museum Avenue

Cardiff CF1 1SS

UK

Keywords: Capsaicin

codeine

cough

cough suppression

naltrexone

Received: July 301993

Accepted after revision December 71993

Opioids possess many pharmacological properties, with analgesia being the most notable. Some opioid substances, such as codeine, are also widely used as antitussive agents in cough medications, and they are believed to exert a relatively specific central inhibitory action on the "cough centre" in the medulla, without causing respiratory depression. When used as an analgesic, codeine behaves as an opioid agonist, and interacts with specific opioid receptor sites in the central nervous system that are also the site of action of a number of endogenous opioid neurotransmitters, such as endorphins and enkephalins [1]. It is reasonable to assume that the antitussive activity of codeine may also be related to this agonist action at central endogenous opioid receptor sites, and that the central component of the cough reflex may be influenced both by opioid agonists and antagonists.

Evidence for the involvement of endogenous opioids in the cough reflex is provided by recent research [2], where it was shown that beta-endorphin, a potent endoge- nous opioid peptide is synthesized in the nucleus tractus solitarius (NTS). The presence of beta-endorphin producing neurons is consistent with the idea that betaendorphins regulate respiration, but the NTS is also the first central synapse of primary afferent fibres that originate from airway receptors [3]. It has been stated [4] that, in addition to regulating respiration, the NTS plays an important role in the regulation of the cough reflex. Thus, endogenous opioids may have a significant antitussive effect, and they may be involved in the voluntary suppression of cough. If this is the case, an opioid antagonist might be expected to alter the action of endogenous opioids on the cough reflex and to enhance cough, especially when these endogenous opioid systems are activated.

In a previous paper [5], we demonstrated that cough induced on inhalation of capsaicin could be voluntarily suppressed, and in the present study, we proposed to investigate the effects of the opioid agonist codeine, and 
the opioid antagonist naltrexone on the voluntary suppression of capsaicin-induced cough. This model of voluntary cough suppression may be particularly useful in studying the effects of an opioid antagonist on cough, as the effects of opioid antagonists on endogenous opioids can only be demonstrated when the endogenous opioid system is activated [1].

The hypothesis to be tested in the present study is that endogenous opioids are involved in the control of capsaicin-induced cough and that an opioid agonist, such as codeine, would be expected to reduce cough, whilst an opioid antagonist, such as naltrexone, would be expected to increase cough, especially during the voluntary suppression of cough.

\section{Subjects and methods}

\section{Subjects}

Volunteers were selected from the student and general population of Cardiff, in response to advertisements for healthy volunteers.

Eighty volunteers presented for the study, 28 males and 52 females, with an average age of 25 yrs (range 18-49 yrs). Subjects read the subject information form, completed a questionnaire concerning their history and signed the informed consent. Each volunteer was then examined by the clinician to determine their state of health and suitability for the trial. Volunteers were fasted on the morning prior to the study.

Volunteers were required to fulfil certain criteria before they were included in the study [5]. Volunteers were also excluded during the study if they failed to cough on capsaicin challenge, or coughed in response to saline (in the baseline non-suppression challenge).

The trial was approved by the local hospital Medical Ethics Committee.

\section{Methods}

The volunteer was seated in a comfortable chair facing a monitor screen. They were instructed to watch the monitor screen carefully and to carry out the instructions as the appropriate box was illuminated. One of two commands was lit up: "Please do not cough" or "Just relax and cough if you wish".

The study was a parallel group design and involved inhalation of single breaths of capsaicin (Cookson Chemicals, UK) in $10 \%$ ethyl alcohol in normal sterile saline solution. Varying concentrations $\left(0.00,1.00 \times 10^{-5}\right.$, $3.33 \times 10^{-5}, 1.00 \times 10^{-4}$ and $\left.3.33 \times 10^{-4} \mathrm{M}\right)$ were delivered by a breath-activated dosimeter (P.K. Morgan, UK). The mass median aerodynamic diameter (MMAD) of the delivered particles was $2-5 \mu \mathrm{m}$. The period of inhalation was set at $1 \mathrm{~s}$. The solutions were made up daily from stock solutions (which were made up weekly), and were stored in a refrigerator at a temperature of $0-6^{\circ} \mathrm{C}$. The nebulized solutions were delivered to the volunteers at room temperature.
Volunteers were given training and instructions as to the appropriate technique for inhalation of the nebulized solutions, and were given a nebulized saline solution on which to practice the technique.

Volunteers received all five concentrations of capsaicin. Each set of five inhalations was defined as a challenge unit. The solutions were administered in random order, with an interval of $60 \mathrm{~s}$ between each inhalation. Two capsaicin challenge units were given $5 \mathrm{~min}$ apart. On one of these challenge units the volunteer was given the instruction "Just relax and cough if you wish", and was able to cough when necessary, and on the other challenge unit, the volunteer was given the instruction "Please do not cough", in which case the volunteer would try to suppress cough for a period of up to $30 \mathrm{~s}$. Volunteers were instructed prior to the study not to hold their breath on this command, and to try to maintain normal breathing. After the $30 \mathrm{~s}$ period of cough suppression the volunteer was given the instruction "Just relax and cough if you wish". The order of the challenge units was randomized between subjects and between treatment groups, i.e. some volunteers were asked to suppress cough on the first challenge unit and some were asked to suppress cough on the second challenge unit.

After receiving two challenge units, each volunteer received a randomized treatment, which consisted of matched capsules of either codeine $(50 \mathrm{mg})$, naltrexone $(50$ $\mathrm{mg}$ ), or placebo. Both the volunteer and the investigator were blind as to which treatment had been administered.

Ninety minutes after administration of the treatment, the volunteer received a further two challenge units of capsaicin. The order of the challenge units was the reverse of that which occurred at baseline.

Coughs were recorded by means of a Sony TCM 84V V.O.R cassette-corder (Taiwan) for a period of $30 \mathrm{~s}$ after each non-suppression challenge and a period of $60 \mathrm{~s}$ after each suppression challenge. Recorded tapes were later played back into a Datagraph (UK) pen recorder, set at a time constant of $0.02 \mathrm{~s}$ to produce integrated pen recorded traces. Coughs were counted from the pen recorder trace according to the method used in a previous study [5].

Volunteers were not permitted to eat or drink during each challenge period, but were asked to drink a glass of water between each challenge unit.

A "Macintosh" "Statview IV" package was used to calculate the statistics. Results are expressed as median (with interquartile range). Nonparametric Freidman tests were used to determine the differences between the three treatment groups at baseline and $90 \mathrm{~min}$ with respect to the number of coughs at each concentration and the total number of coughs in each challenge unit.

Changes in cough response due to codeine, naltrexone or placebo were obtained by subtracting the baseline total cough response (i.e. the sum of the coughs from all capsaicin inhalations in a challenge unit) from the total number of coughs $90 \mathrm{~min}$ after treatment. Changes in the cough responses were obtained for the non-suppression and suppression challenges. Wilcoxon signed rank tests were then used to determine differences within each 
treatment group between the baseline and 90 min challenges. Values of $\mathrm{p}<0.05$ were considered to be significant. A retrospective power calculation at a power level of $80 \%$ (5\% significance level) found that 27 subjects were required in each group to detect a $30 \%$ change in total cough counts.

\section{Results}

From the 80 subjects entered into the study, seven were excluded from the analysis. Five of these subjects had a total cough count of $\geq 30$ at baseline and were, therefore, considered to be hypersensitive to capsaicin. The remaining two subjects suffered from nausea and vomiting as a result of treatment (1 codeine, 1 naltrexone) and were withdrawn from the study prior to the $90 \mathrm{~min}$ challenge. Of the remaining 73 subjects, the distribution of the treatments was: codeine: 27 (at baseline 14 received non-suppression challenge first and 13 received suppression challenge first); naltrexone: 25 (14 nonsuppression first, 11 suppression first); and placebo: 21 (10 non-suppression first and 11 suppression first).

\section{Non-suppression capsaicin challenge}

At baseline, a cough dose response relationship was found on administration of increasing concentrations of capsaicin in response to the instruction "Just relax and cough if you wish". These results correspond to those obtained in a previous study [5]. Similarly, 90 min after treatment, dose response relationships were again seen.

There was no significant difference between any of the three treatment groups at any concentration of capsaicin at baseline ( $p$ corrected for ties $>0.48$ for all concentrations), or $90 \mathrm{~min}$ after treatment ( $\mathrm{p}$ corrected for ties $>0.18$ for all capsaicin concentrations).

Total coughs were calculated for each subject in the three treatment groups by adding the number of coughs produced at each concentration of capsaicin in the nonsuppression challenge. Table 1 shows the median total number of coughs at baseline and 90 min after treatment in the non-suppression challenge in the three treatment groups. No significant difference was found for the total number of coughs between the three treatment groups at baseline ( $\mathrm{p}$ corrected for ties $=0.81$ ), or at $90 \mathrm{~min}$ ( $\mathrm{p}$ corrected for ties $=0.78$ ).

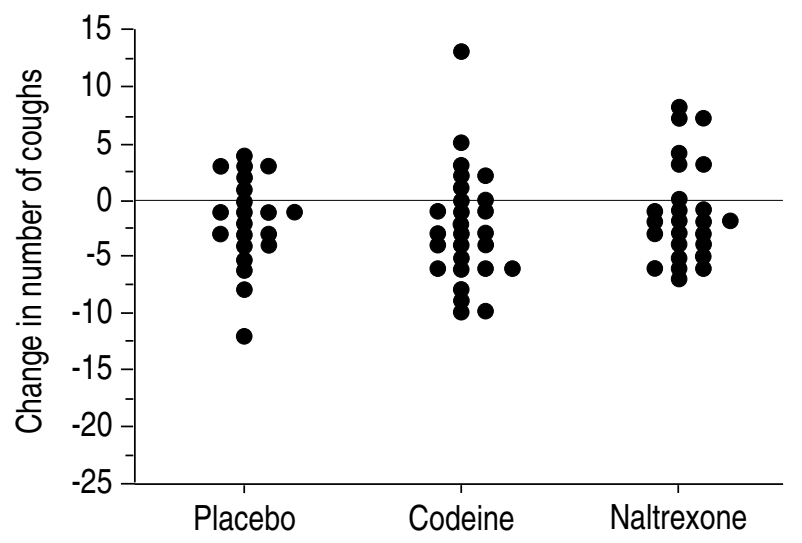

Fig. 1. - Changes in the number of coughs to capsaicin after treatment in the non-suppression challenge. Data represent the changes in cough response in the placebo, codeine or naltrexone treated groups, obtained by subtracting the total number of coughs in the nonsuppression challenge at baseline from the total number of coughs in the non-suppression challenge $90 \mathrm{~min}$ after treatment.

\section{Changes in cough response following treatment}

Changes in cough responses between the baseline and treatment non-suppression challenges were calculated for the placebo, codeine and naltrexone groups and are illustrated in figure 1. The total number of coughs at $90 \mathrm{~min}$ was generally less than that at baseline in all three treatment groups as shown in table 1 . There were 14 out of 21 placebo, 19 out of 27 codeine and 18 out of 25 naltrexone subjects who showed a reduction in the total number of coughs in the non-suppression challenge 90 min after treatment, compared to baseline. Wilcoxon signed rank tests were used to compare the total number of coughs at baseline and $90 \mathrm{~min}$ in each of the treatment groups, and tied p-values were 0.07, 0.01 and 0.17 for placebo, codeine and naltrexone, respectively. Codeine was the only treatment group which showed a significant reduction from baseline; however, when this was compared across groups there was no significant difference between codeine and placebo groups.

\section{Suppression capsaicin challenge}

Following the instruction "Please do not cough", the number of coughs both at baseline and 90 min during

Table 1. - Median total number of coughs (with interquartile range in parenthesis) for each treatment group

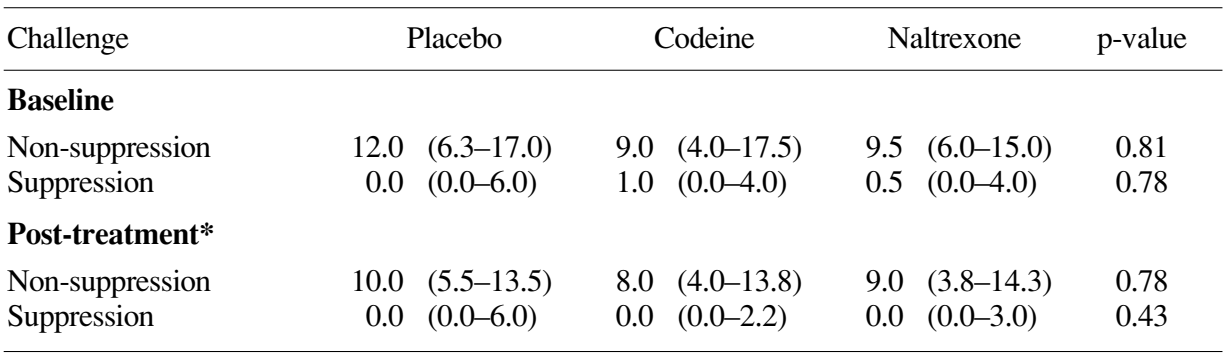

Total coughs were calculated by adding the number of coughs produced at each concentration of capsaicin within the different challenge units. *: 90 mins post-treatment. 


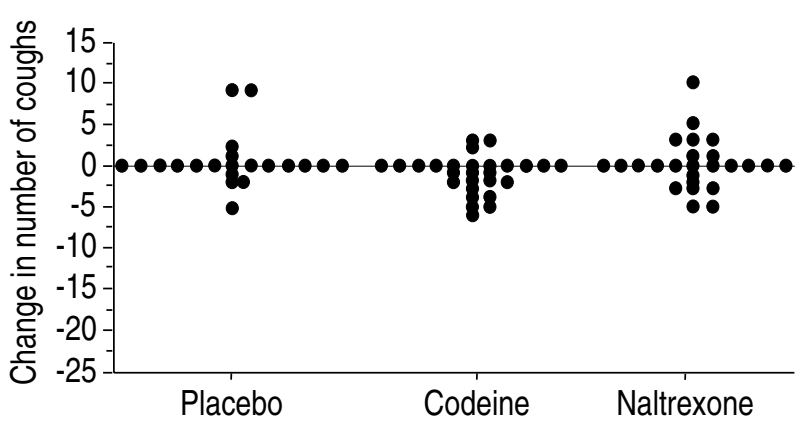

Fig. 2. - Changes in the number of coughs to capsaicin after treatment in the suppression challenge. Data represent the changes in cough response in the placebo, codeine or naltrexone treated groups and were obtained by subtracting the total number of coughs in the suppression challenge at baseline from the total number of coughs in the suppression challenge $90 \mathrm{~min}$ after treatment.

the suppression challenge was significantly reduced in all three treatment groups, compared to those recorded in the non-suppression challenge (tied $\mathrm{p}<0.01$ for all capsaicin concentrations, Wilcoxon signed rank test). There was no significant difference between the three treatment groups at any concentration of capsaicin in the suppression challenge at baseline ( $\mathrm{p}$ corrected for ties $>0.34$ for all capsaicin concentrations), or $90 \mathrm{~min}$ after treatment ( $p$ corrected for ties $>0.37$ for all capsaicin concentrations). The majority of subjects (41 out of 73 at baseline, 48 out of 73 at $90 \mathrm{~min}$ ) completely suppressed cough at the highest capsaicin concentration. Table 1 shows the median total number of coughs during the suppression challenge at baseline and $90 \mathrm{~min}$ after treatment, and shows that there was no significant difference in the total number of coughs between the three treatment groups ( $p$ corrected for ties $=078$ at baseline, and $0.4390 \mathrm{~min}$ after treatment).

\section{Changes in the cough response following treatment}

Changes in the cough response for each of the three treatment groups during the suppression challenge are illustrated in figure 2. As with the non-suppression challenge, the total number of coughs in the suppression challenge was generally less at $90 \mathrm{~min}$ than at baseline. In the placebo group, 4 out of 21 subjects showed a reduction in the number of coughs compared with 13 out of 27 in the codeine group and 7 out of 25 in the naltrexone group. A large number of subjects (35 out of 75) showed no change in cough count from baseline. Using the Wilcoxon signed rank test, it was found that there was no significant difference between the total number of coughs at baseline and $90 \mathrm{~min}$ after treatment in any of the three treatment groups during the suppression challenge (tied $\mathrm{p}$-value $=0.78,0.14$ and 0.93 for placebo, codeine and naltrexone, respectively).

\section{Discussion}

The results demonstrate that cough induced by inhalation of capsaicin can be voluntarily suppressed with the majority of subjects (41 out of 73) completely suppressing cough at the highest concentration of capsaicin $\left(3.33 \times 10^{-4} \mathrm{M}\right)$ in this study.

The present study was a parallel group design, and all three treatment groups were well-balanced before treatment with no significant difference between the three groups for any of the cough challenges. Following treatment with placebo, codeine $(50 \mathrm{mg})$ or naltrexone $(50$ $\mathrm{mg}$ ), there was no significant difference between the three treatment groups when comparing the median coughs at each concentration of capsaicin and the median total number of coughs for each challenge unit.

When comparisons were made within groups before and after treatment, only the codeine group showed a significant reduction in the cough response for the nonsuppression challenge. However, this effect of codeine was very small, and when comparisons were made across groups there was no significant difference between the three groups. Some reduction in cough response due to a placebo effect and due to training and adaptation to the cough stimulus would be expected, and this might account for the significant result obtained after treatment with codeine. Capsaicin-induced cough may also be subject to tachyphylaxis for long periods of inhalation up to 1 min [6], but this is not a significant effect in relation to the short periods of inhalation used in this study [7]. Similarly, there should be no carry-over effects of successive within-days challenges, since we have shown in a previous study (Hutchings et al. unpublished data) that there was no significant difference between challenges performed on the same day. However, since the results were compared across groups (i.e. with placebo), any tachyphylaxis or carry-over effects would be controlled.

The failure to show any significant difference between the three groups on capsaicin challenge does not support the hypothesis that the opioid agonist codeine would reduce cough and the opioid antagonist naltrexone would increase cough due to the involvement of endogenous opioids in the cough reflex.

In a study of this kind, it is impossible to determine how voluntary suppression of cough occurs. It may be that the cough reflex is suppressed secondarily to an altered respiratory pattern. This seems unlikely, however, since volunteers were instructed not to hold their breath and to maintain a normal breathing pattern. Since respiratory rhythm was not monitored, however, we cannot be completely sure that normal respiration was maintained. In addition, there appears to be no evidence that altered blood gases or respiratory rates have any effect on the cough reflex. Similarly, if the treatments used affected the respiratory centres, we may have been more likely to see varying results in the different treatment groups. It has also been stated [8] that it is unlikely that coughing is depressed by an action on the respiratory centres themselves, and it was demonstrated that anaesthesia strongly inhibited respiration without blocking the cough reflex, whereas, antitussives could prevent coughing with little respiratory depression.

A negative result is always difficult to interpret, as it is necessary to ensure that the methodology of the study is sufficiently reliable to adequately test the hypothesis 
under scrutiny. Capsaicin inhalation was used to produce cough, and although it could be argued that different peripheral sensory receptors are involved from those in natural cough, the central control mechanism for the cough reflex is believed to be the same for all types of cough. If opioid agonists are believed to inhibit cough by a central inhibition, one would expect all forms of cough to be affected. The lack of effect of codeine and naltrexone in the present study cannot, therefore, be explained by some mechanism of cough which is specific to capsaicin.

The doses of the drugs used in this study were above the usual therapeutic doses, the subjects were fasted prior to treatment, and cough measurements were taken 90 min after treatment to ensure maximal plasma drug levels for both drugs. Indeed, two volunteers were excluded from the study analysis due to nausea and vomiting. This indicates that the drugs were adequately absorbed and were acting at the limit of the therapeutic range. The negative result cannot, therefore, be explained by inadequate dosing or absorption of the drug treatments.

Coughs were measured in the present study as frequencies, and although neither codeine nor naltrexone showed any change in cough frequency after treatment, it is possible that the drugs influenced some other cough parameter, such as latency or intensity.

Single-breath inhalations of capsaicin were used in this study, and this causes an immediate short-lived irritation of receptors. Since the effect was very rapid, it may be insufficient to activate the endogenous opioid systems. Thus, no effect would be seen after naltrexone treatment. When endogenous opioid systems are activated, the effects of opioid antagonists on endogenous opioids become measurable [1]. It may, therefore, be possible to design a future study, whereby inhalations of capsaicin over longer periods are used, or to study the effects of opioid agonists and antagonists on cough resulting from acute upper respiratory tract infection, where an ongoing or more prolonged receptor irritation is seen.

It may be that endogenous opioids are not involved in cough suppression, and that other CNS neurotransmitters, such as serotonin or gamma-amino butyric acid (GABA)-b agonists are involved [9-11]. Again, this introduces a whole new area of study.

Further studies are required to examine our hypothesis in more detail. But, at present, the results of this study seem to indicate that opioid systems are not acti- vated in the voluntary suppression of capsaicin-induced cough, and that both codeine, a well-established antitussive treatment acting as an opioid agonist, and naltrexone, an opioid antagonist, have no effect on the cough reflex or the ability to voluntarily suppress cough induced by capsaicin inhalation.

Acknowledgement: The authors would like to thank Procter and Gamble for sponsoring this project and H.W. Thomas for his help and advice on the statistical analysis of the data.

\section{References}

1. Jaffe JH, Martin WR. Opioid analgesics and antagonists. In: Gilman A, Goodman LS, Rall W, Murad F, eds. Goodman and Gilman's: The Pharmacological Basis of Therapeutics. New York, Macmillan Publishing Co., 1985; Chapter 22: pp. 491-531.

2. Bronstein DM, Schafer MKH, Watson SJ, Akul H. Evidence that beta-endorphin is synthesized in cells in the nucleus tractus solitarius: detection of POMC mRNA. Brain Res 1992; 587: 269-275.

3. Kamei J, Iwamoto Y, Misawa M, Nagase H, Kasuya Y. Antitussive effect of beta-endorphin is mediated by mu-opioid receptors, but not by kappa or epsilon-opioid receptors. Eur J Pharmacol 1993; 233: 251-254.

4. Korpas J, Tomori Z. Cough and other respiratory reflexes. In: Progress in Respiration Research. Basel, Karger, 1979; Vol. 12: pp. 15-188.

5. Hutchings HA, Morris S, Eccles R, Jawad MSM. Voluntary suppression of cough induced by inhalation of capsaicin in healthy volunteers. Respir Med 1993; 87: 379-382.

6. Morice AH, Higgins KS, Yeo WW. Adaptation of cough reflex with different types of stimulation. Eur Respir $J$ 1992; 5: 841-847.

7. O'Connell F, Thomas VE, Pride NB. Adaptation of cough reflex with different types of stimulation (Correspondence). Eur Respir J 1992; 5: 1296-1297.

8. May AJ, Widdicombe JG. Depression of the cough reflex by pentobarbitone and some opium derivatives. $B r J$ Pharmacol 1954; 9: 335-340.

9. Bolser DC, Aziz SM, DeGennaro FC, et al. Antitussive effects of $\mathrm{Gaba}_{\mathrm{b}}$ agonists in the cat and guinea-pig. $\mathrm{Br}$ J Pharmacol 1993; 110: 491-495.

10. Bolser DC, Aziz SM, Kreutner W, Chapman RC. Baclofen has antitussive effects in the cat. FASEB J 1991; 5: A1244.

11. Stone RA, Wordsell YM, Fuller RW, Barnes PJ. Effects of 5- hydroxytryptamine and 5-hydroxytryptophan infusion on the human cough reflex. J Appl Physiol 1993; 74(1): $396-401$. 